\title{
Detection of adenovirus E1A DNA in pulmonary fibrosis using nested polymerase chain reaction
}

\author{
K. Kuwano, Y. Nomoto, R. Kunitake, N. Hagimoto, T. Matsuba, Y. Nakanishi, N. Hara
}

Detection of adenovirus E1A DNA in pulmonary fibrosis using nested polymerase chain reaction. K. Kuwano, Y. Nomoto, R. Kunitake, N. Hagimoto, T. Matsuba, Y. Nakanishi, N. Hara. @ERS Journals Ltd 1997.

ABSTRACT: The history of patients with idiopathic pulmonary fibrosis (IPF) shows that the disease may be preceded by a viral-like illness. Although viruses have not been demonstrated, it is possible that viruses were not detected in culture because they do not replicate during latency.

We investigated the presence of adenovirus in IPF and interstitial pneumonia associated with collagen vascular disease (CVD-IP), using the nested polymerase chain reaction (PCR) and in situ hybridization (ISH) for the E1A region of the adenovirus genome. Studies were performed on lung tissues obtained by transbronchial lung biopsy from 19 patients with IPF, 10 patients with CVD-IP and, for comparison, from 20 patients with sarcoidosis.

The E1A DNA was present in 3 out of $19(16 \%)$ cases of IPF, in 5 of $10(50 \%)$ cases of CVD-IP, and in 2 of $20(10 \%)$ cases of sarcoidosis. The incidence of E1A DNA in CVD-IP was significantly higher than that in sarcoidosis $(p<0.05)$. In patients with IPF and CVD-IP, E1A DNA was more prevalent in patients treated with corticosteroids ( 6 out of 9 cases; $67 \%$ ) than in those without it ( 2 out of 20 cases; $10 \%)(\mathrm{p}<0.01)$. ISH studies showed that 1 out of 8 cases of IPF and CVD-IP, in which E1A DNA was detected by PCR, was positive for E1A DNA.

We conclude that adenovirus E1A is unlikely to be aetiologically involved in the pathogenesis of idiopathic pulmonary fibrosis or interstitial pneumonia associated with collagen vascular disease. However, a latent adenovirus infection may be reactivated or may newly infect the host following corticosteroid administration. Eur Respir J 1997; 10: 1445-1449.
Research Institute for Diseases of the Chest, Faculty of Medicine, Kyushu University, 3-1-1 Maidashi, Higashiku, Fukuoka 812, Japan.

Correspondence: K. Kuwano

Research Institute for Diseases of the Chest Faculty of Medicine

Kyushu University

3-1-1 Maidashi

Higashiku

Fukuoka 812

Japan

Keywords: Adenovirus polymerase chain reaction pulmonary fibrosis

Received: April 211995

Accepted after revision December 101996
In vitro deoxyribonucleic acid (DNA) amplification by the polymerase chain reaction (PCR) makes it possible to detect rare DNA sequences [1], and can be used to identify the nucleic acids of infectious agents present in copy numbers that are too low to be detected by other methods [2]. This sensitive detection system has also been used for the identification of viral DNA in archival material, such as paraffin-embedded fixed tissue, where much of the DNA has been degraded and is no longer suitable for conventional methods of DNA analysis [3].

Although no one has been able to demonstrate viruses in the bronchoalveolar lavage fluid or in lung tissues from patients with idiopathic pulmonary fibrosis (IPF), it is possible that viruses were not detected in culture because they do not replicate during latency. Latent viruses may produce viral proteins that modulate viral and host immune responses and can perhaps be reactivated by a stimulus, such as immunosuppression.

Infection by group $\mathrm{C}$ adenovirus (types 1, 2, 5 and 6) is common in humans, causing colds and other types of infections. These viruses tend to become latent in the tonsils [4], the peripheral lymphocytes [5], and the lung [6]. The E1A region of the adenovirus was used as the target DNA for amplification in this study because this region of the virus is responsible for the transformation of the cells and for sensitizing the infected cells to cytolysis by tumour necrosis factor (TNF) [7], natural killer (NK) cells, and activated macrophages [8]. If adenovirus is present, it could cause or modulate alveolitis, which is thought to be the initial phenomenon in pulmonary fibrosis. Patients with IPF and interstitial pneumonia associated with collagen vascular disease (CVD-IP) were often treated with corticosteroid and/or other immunosuppressive agents. We hypothesized that latent adenovirus infection may cause or modulate the pathophysiology of pulmonary fibrosis, and that the virus may be reactivated by immunosuppressive therapy.

To investigate this hypothesis, we used nested PCR, which is a two-step in vitro amplification, first with outer primers and then with inner primers nested within the outer primers, attempting to increase both the sensitivity and the specificity of the PCR [9], to determine the incidence of adenovirus DNA in lung tissues from patients diagnosed as IPF or as CVD-IP. We also used an in situ hybridization (ISH) method to localize the adenovirus DNA in specific lung cells. 


\section{Materials and methods}

\section{Case material}

The study of IPF was performed on 22 samples of lung tissues obtained by transbronchial lung biopsy (TBLB) from 19 patients with IPF, 8 males and 11 females, aged 41-81 yrs (mean 62 yrs). There were 10 smokers and 9 nonsmokers. The diagnosis of IPF was established by a combination of medical history, physical examination, laboratory tests, chest radiography, pulmonary function tests, and the results of pulmonary biopsies, according to criteria described previously [10].

The study of CVD-IP was performed on 17 samples of lung obtained by TBLB from 10 patients. There were 4 males and 6 females, aged 37-67 yrs (mean 56 yrs). Three patients were smokers and seven patients were nonsmokers. There were five cases of rheumatoid arthritis, two cases of dermatomyositis with Sjögren's syndrome, one case of rheumatoid arthritis with progressive systemic sclerosis, one case of dermatomyositis, and one case of progressive systemic sclerosis. The diagnosis of collagen vascular disease was based on clinical criteria, using a combination of medical history, physical examination, laboratory tests, and chest radiography. Patients with collagen vascular diseases were all in the chronic stage, and pathological findings exhibited the usual features of fibrosing alveolitis of the usual interstitial pneumonia variety. More than three specimens from different parts of the lung were obtained by TBLB. Specimens that consisted of bronchial mucosa only were excluded. We ensured that the specimens included bronchiolar and alveolar epithelial cells.

Results in these two groups (IPF and CVD-IP) were compared with those in patients with sarcoidosis, in which 20 samples of lung tissues were obtained from 20 patients with sarcoidosis by TBLB. There were 12 males and 8 females, aged 23-60 yrs (mean 42 yrs). The diagnosis of sarcoidosis was confirmed by the presence of noncaseous epithelioid granulomas in tissue samples.

\section{DNA extraction}

Specimens were obtained by TBLB, fixed in $10 \%$ formalin, and routinely embedded in paraffin. Four $10 \mu \mathrm{m}$ sections were cut from each block, using a fresh disposable microtome blade for each block to prevent crosscontamination. Sections were rinsed with xylene to remove paraffin and washed with ethanol. Tissue was suspended in $200 \mu \mathrm{L}$ of a mixture of $100 \mathrm{mM}$ Tris $\mathrm{HCl}(\mathrm{pH}$ 8.0); $40 \mathrm{mM} \mathrm{Na}_{2}$ ethylenediamine tetra-acetic acid (EDTA);
$10 \mathrm{mM} \mathrm{NaCl} ; 1 \%$ sodium dodecyl sulphate (SDS) and proteinase K (Amresco Inc., Solon, OH, USA), 500 $\mu \mathrm{g} \cdot \mathrm{mL}^{-1}$. Samples were incubated at $50^{\circ} \mathrm{C}$ overnight. This mixture was subjected to phenol/ $\mathrm{CHCl}_{3}(1: 1)$ extraction. After ethanol precipitation, the DNA was dried.

\section{Oligonucleotide primers}

The nucleotide sequences of the primers and probes are presented in table 1 . The primers used in the first PCR specified the amplification of a 675 base pair (bp) fragment of the E1A region of the genome of adenovirus 5 [6]. This primer pair also amplified a $673 \mathrm{bp}$ fragment from the corresponding region of adenovirus 2. The primers used in the nested PCR specified the amplification of a $261 \mathrm{bp}$ fragment of the inner part of the first PCR product. The primers used to confirm the presence of DNA extracted from paraffin-embedded tissues specified the amplification of a $240 \mathrm{bp}$ fragment of the human leucocyte antigen (HLA)-DQ $\alpha$ gene [11].

\section{Polymerase chain reaction}

The extracted DNA was dissolved in a solution containing: $10 \mathrm{mM}$ Tris $\mathrm{HCl}(\mathrm{pH} 8.4) ; 50 \mathrm{mM} \mathrm{MgCl}$; $0.01 \%$ Triton X-100; $100 \mu \mathrm{M}$ each of deoxyguanosine triphosphate (dGTP), deoxyadenosine triphosphate (dATP), deoxycytidine triphosphate (dCTP), deoxythymidine triphosphate (dTTP); and $0.5 \mu \mathrm{M}$ each of the first set of primers. After the mixture had been heated in a DNA thermal cycler (Perkin-Elmer Cetus, Emoryville, CA, USA) at $100^{\circ} \mathrm{C}$ for $10 \mathrm{~min}, 2.5$ units of Taq DNA polymerase (Takara Biochemicals, Otsu, Japan) were added. The entire mixture of $50 \mu \mathrm{L}$ was overlaid with $40 \mu \mathrm{L}$ of mineral oil and subjected to 35 cycles of 1 min denaturation at $93^{\circ} \mathrm{C}, 1 \mathrm{~min}$ to allow annealing at $63^{\circ} \mathrm{C}$, and 2 min of extension at $72^{\circ} \mathrm{C}$. During the last cycle, the extension time was increased by $7 \mathrm{~min}$. Five microlitres of the first PCR product was subjected to nested PCR, which differed from the first PCR only in the primers used. The signal of the PCR product from each block was analysed by electrophoresis in $2 \%$ agarose gel.

The reaction conditions for amplification of the HLADQ $\alpha$ gene were the same as those for the E1A PCR, except that the $\mathrm{MgCl}_{2}$ concentration was $2.5 \mathrm{mM}$. The thermocycler programme was the same as that described above, except that the annealing step was for $2 \mathrm{~min}$ at $45^{\circ} \mathrm{C}$. The DNA was transferred from the gel to the filter. Filters with HLA-DQ $\alpha$ PCR products were prehybridized and hybridized with oligonucleotide probes labelled with digoxigenin-dideoxyuridine triphosphate

Table 1. - Oligonucleotides used in polymerase chain reaction analysis

\begin{tabular}{ll}
\hline Primer or probe & Oligonucleotide sequence \\
\hline E1A outer primers & 5'-CTGCCACGGAGGTGTTATTACC-3' \\
& 5'-CTCAGGTTCAGACACAGGACCT-3' \\
E1A inner primers & 5'-GAACCACCTACCCTTCACGAACTG-3' \\
& 5'-GTGGCAGGTAAGATCGATCACCTC-3' \\
HLA-DQ $\alpha$ primers & 5'-GTGCTGCAGGTGTAAACTTGTACCAG-3' \\
HLA-DQ $\alpha$ probe & 5'-GTTGAGATGGCGACGATGGCCTAGGCAC-3' \\
\end{tabular}

HLA: human leucocyte antigen; C: cytosine; T: thymine; G: guanine; A: adenine. 
(ddUTP) using the DIG Oligonucleotide 3'-End Labeling Kit (Boehringer Mannheim, Mannheim, Germany). Hybridization was performed according to protocols recommended by the manufacturer, and the digoxigeninlabelled probe hybridized with HLA-DQ $\alpha$ PCR products was detected by means of the DIG Nucleic Acid Detection Kit (Boehringer Mannheim).

All samples were tested simultaneously, along with positive and negative controls. For positive controls, serially diluted adenovirus 2 DNA and DNA extracted from paraffin sections of fixed Graham 293 cells, which contain 4-5 copies of the E1A region of adenovirus 5 [12], were used. Human placental DNA (250 ng) and no template DNA, which consisted of a sample containing all the components for PCR except template DNA, served as negative controls. DNA extracted from Raji cells, that were fixed and embedded in paraffin, was used as an additional negative control in the series of paraffin-embedded lung tissues. Human placental DNA (250 ng) and no template DNA served as positive and negative controls, respectively, for HLA-DQ $\alpha$ amplification.

\section{In Situ hybridization}

Two 5 um sections were cut from each block, and placed on opposite ends of silane-coated slides. These sections were dewaxed by washing for $5 \mathrm{~min}$ three times in xylene, dehydrated in 100,95 and $80 \%$ ethanol for 5 min each, and allowed to dry. They were then incubated in acid pepsin solution (porcine stomach mucosa pepsin (Sigma Chemical Co., St. Louis, MO, USA), 1.0 $\mathrm{mg} \cdot \mathrm{mL}^{-1}$ in distilled water $\left(\mathrm{dH}_{2} \mathrm{O}\right)$ acidified to $0.2 \mathrm{M}$ $\mathrm{HCl}$ ) for $10 \mathrm{~min}$, and immediately fixed in $4 \%$ paraformaldehyde at room temperature for $5 \mathrm{~min}$. The slides were then washed with $0.5 \%$ Triton $\mathrm{X}-100$ in phosphate-buffered saline (PBS) for $5 \mathrm{~min}$ at room temperature, and incubated in $1 \%$ glycine-PBS solution. After being rinsed with $\mathrm{dH}_{2} \mathrm{O}$, the slides were dehydrated in 80,95 and $100 \%$ ethanol and allowed to dry. One section was inverted onto a coverslip with $10 \mu \mathrm{L}$ of hybridization solution containing biotinylated adenovirus 5 probe (Enzo Diagnostics, New York, USA). The other section on the same slide was inverted onto another coverslip with $10 \mu \mathrm{L}$ of hybridization solution containing biotinylated plasmid pBR322 DNA (Enzo Diagnostics).

The DNA in both probe and tissue was denatured at $96^{\circ} \mathrm{C}$ for $10 \mathrm{~min}$, and allowed to hybridize for $3 \mathrm{~h}$ at $37^{\circ} \mathrm{C}$ in humidified plastic dishes. The coverslips were then rinsed with $2 \times$ standard sodium citrate (SSC), and the slides were washed twice in $2 \times$ SSC for 5 min each time, twice in $0.1 \times \mathrm{SSC}$ for $5 \mathrm{~min}$ each time, and washed once in $2 \times \mathrm{SSC}$ for $2 \mathrm{~min}$ at room temperature. Five hundred microlitres of $3 \%$ bovine serum albumin (BSA) in $0.5 \%$ Triton X-100 PBS solution was placed on the sections, and the slides were then incubated for $10 \mathrm{~min}$ at $37^{\circ} \mathrm{C}$. The slides were washed with PBS, blotted to remove excess moisture, and incubated in the same BSA solution containing the streptavidin-alkaline phosphatase conjugate (Bethesda Research Laboratories, Gaithersburg, MD, USA) at a concentration of $1 \mathrm{U} \cdot \mathrm{mL}^{-1}$, for $30 \mathrm{~min}$ at $37^{\circ} \mathrm{C}$. After being rinsed with PBS, the slides were washed three times (for $5 \mathrm{~min}$ each time) in 0.5 $\%$ Triton/PBS, three times (for 5 min each time) in PBS, and three times (for 5 min each time) in AP $9.6(0.1 \mathrm{M}$ Tris $\mathrm{HCl}(\mathrm{pH} 9.6), 0.1 \mathrm{M} \mathrm{NaCl}, 0.1 \mathrm{M} \mathrm{MgCl}_{2}$ ). They were then placed in AP 9.6 containing 5-bromo-4-chloro3 -indole phosphate, $169 \mu \mathrm{g} \cdot \mathrm{mL}^{-1}$, (Sigma), and nitro blue tetrazolium, $331 \mu \mathrm{g} \cdot \mathrm{mL}^{-1}$ (Sigma), and left in the dark for $30 \mathrm{~min}$. The colour reaction was stopped by washing with $\mathrm{Na}_{2}$ EDTA, and the slides were examined under a light microscope. Slides prepared from formalin-fixed, paraffin-embedded lung tissue obtained from a patient with adenoviral pneumonia served as a positive control for the detection of viral DNA. The negative control for the detection system was obtained by comparing the section treated with the adenoviral DNA probe with an adjacent section on the same slide treated with pBR322 DNA.

\section{Statistical methods}

For PCR analysis, the incidence of E1A DNA in the IPF, CVD-IP, and sarcoidosis groups was analysed by Chi-squared test. A p-value of less than 0.05 was considered statistically significant.

\section{Results}

Figure 1 presents a representative electrophoresed agarose gel of the products of PCR amplification for serially-diluted adenoviral DNA and positive and negative controls. In the nested PCR, one copy of adenoviral DNA and DNA extracted from formalin-fixed, paraffinembedded Graham cells were successfully amplified. The negative controls, consisting of human placental DNA and DNA extracted from histological sections of Raji cells, did not show a visible amplification product.

a)
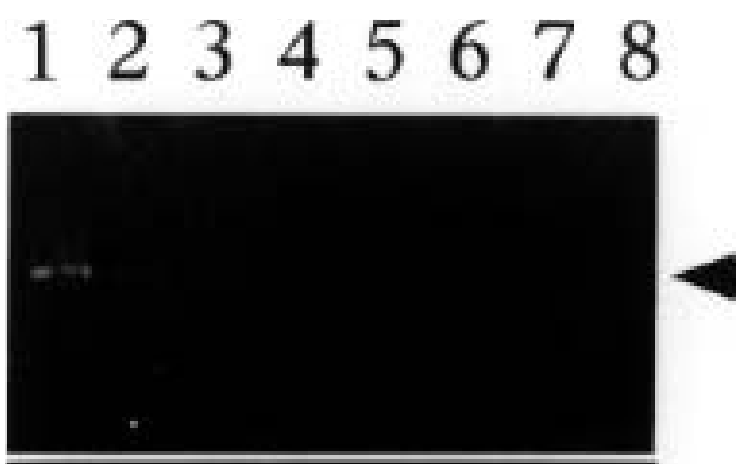

b)

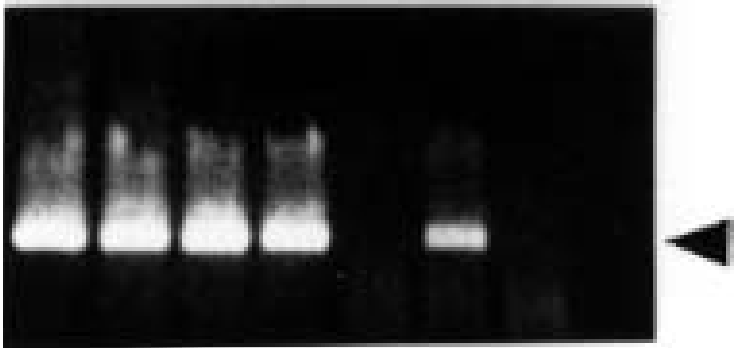

Fig. 1. - a) Conventional; and b) nested polymerase chain reaction (PCR) amplification of adenovirus deoxyribonucleic acid (DNA). Agarose gel of PCR amplification products. The template DNAs used in the PCRs were the following: lanes 1 to 4, DNA from serially diluted adenovirus DNA $\left(10^{3}, 10^{2}, 10^{1}, 10^{0}\right.$ copies $)$; lane 5 , no template DNA; lane 6, DNA from Graham cells; lane 7, DNA from Raji cells; lane 8 , human placental DNA. 
Table 2. - Summary of results of PCR analysis

\begin{tabular}{|c|c|c|c|}
\hline \multicolumn{4}{|c|}{ E1A PCR positive } \\
\hline & IPF & CVD-IP & Sarcoidosis \\
\hline & $\mathrm{n} \%$ & $\mathrm{n} \%$ & $\mathrm{n} \quad \%$ \\
\hline Cases & $3 / 19(16)$ & $5 / 10(50) *$ & $2 / 20(10)$ \\
\hline Blocks & $4 / 22$ (18) & $6 / 17(35)$ & $2 / 20(10)$ \\
\hline \multicolumn{4}{|c|}{ E1A PCR positive in IPF and CVD-IP } \\
\hline & Steroid $(+)$ & & Steroid(-) \\
\hline & $\mathrm{n} \quad \%$ & & \\
\hline Cases & $6 / 9(67)^{\dagger}$ & & $2 / 20(10)$ \\
\hline Blocks & $6 / 10(60)^{\dagger}$ & & $4 / 29(14)$ \\
\hline
\end{tabular}

PCR: polymerase chain reaction; IPF: idiopathic pulmonary fibrosis; CVD-IP: interstitial pneumonia associated with collagen vascular disease; Steroid(+); patients administered corticosteroids; Steroid(-): patients not treated with corticosteroids. *: CVD-IP > sarcoidosis, $\mathrm{p}<0.05$; ${ }^{\dagger}$ Steroid(+) > Steroid(-), $\mathrm{p}<0.01$.

Table 2 summarizes the results of PCR analysis. Adenoviral DNA was found in 3 out of $19(16 \%)$ patients with IPF, in 5 of $10(50 \%)$ patients with CVD-IP, and in 2 of $20(10 \%)$ patients with sarcoidosis. The prevalence of adenovirus in CVD-IP was significantly higher than that in sarcoidosis $(\mathrm{p}<0.05)$. In the IPF and CVD-IP patients, 6 out of 8 cases $(75 \%)$, that were positive for adenovirus DNA by PCR, corticosteroids were administered for 1-18 months. The incidence of adenovirus DNA in patients with IPF and CVD-IP who received corticosteroids was 6 out of 9 cases $(67 \%)$, which was significantly higher $(\mathrm{p}<0.01)$ than that in patients who did not have corticosteroid treatment $(2$ out of 20 cases; 10\%). The incidence of adenovirus DNA in smokers (3 out of $13 ; 23 \%$ ) did not differ from that in nonsmokers $(5$ out of $16 ; 31 \%$ ) in IPF and CVDIP patients. Hybridization of HLA-DQ $\alpha$ PCR products with an oligonucleotide probe confirmed the successful extraction of DNA from all samples used.

ISH analysis was performed on sections cut from all blocks used for PCR. Although 10 cases, which consisted of three cases of IPF, five cases of CVD-IP, and two cases of sarcoidosis, were positive for E1A by PCR as shown in table 2, only one case of CVD-IP showed positive signals for adenovirus probe in alveolar epithelial cells by ISH. All the cases negative for E1A PCR were also negative for adenovirus DNA by ISH.

\section{Discussion}

In this study, the negative E1A PCR controls, the samples without added template DNA, the samples with purified human placental DNA, and the samples with DNA extracted from paraffin-embedded Raji cells, all of which were analysed at the same time as DNA from the test samples, were consistently negative. Thus, we are confident that our results are reliable and unaffected by contamination. Figure 1 shows clearly that nested PCR for E1A DNA was at least 100 times more sensitive than conventional PCR, and able to detect a single copy of adenovirus DNA. The PCR product was visualized by agarose gel electrophoresis and ethidium bromide staining. Thus, we were able to avoid the need for hybridization in order to detect the PCR product.

Transbronchial biopsy in IPF is often inadequate, ten- ding to sample endobronchial tissues only. We performed TBLB until we obtained at least three specimens. Only specimens that included adequate lung parenchyma, bronchiolar and alveolar epithelial cells, and lymphocytes were used. Thus, the TBLB specimens used in this study were adequate for PCR study of adenovirus DNA.

The possible role of infection in the genesis of pulmonary fibrosis is often questioned [13]. In a review of IPF, some $25-40 \%$ of patients with IPF reported clearly that their disease began after a viral syndrome that included chest symptoms [10]. Although no virus has been cultured in IPF, the virus could be latent and, thus, it would not replicate. The techniques of molecular biology now make it possible to detect a latent virus infection in clinical specimens that was not previously detectable by culture. Previous study [6] showed that the PCR technique demonstrated small amounts of adenovirus DNA that are commonly present in human lungs.

We observed that adenovirus was more prevalent in lung tissues obtained from patients treated with corticosteroids (67\%) than in those who were not (10\%) (table 2 ). The incidence of adenovirus in cases of pulmonary fibrosis without corticosteroid therapy was identical to that in patients with sarcoidosis $(10 \%)$, who were not administered corticosteroids before biopsy. It therefore seems unlikely that adenovirus infection is directly involved in the pathogenesis of pulmonary fibrosis. MATSuse et al. [6] demonstrated that adenovirus E1A DNA is positive in $87 \%$ of paraffin-embedded specimens of lung parenchyma obtained from patients who underwent lobectomy for lung carcinoma. In a study involving fresh blocks of lung parenchyma from smokers, the incidence of E1A positivity was $44 \%$ in patients without chronic obstructive pulmonary disease (COPD), and $90 \%$ in those with COPD [6]. Thus, in the present study, the frequency of positivity for adenovirus in pulmonary fibrosis without corticosteroid treatment (10\%) was less than that in normal lung parenchyma in the previous study [6], and even the high values in corticosteroid-treated patients $(67 \%)$ was no higher than that in normal lung parenchyma.

BATEMAN et al. [14] reported that the incidence of adenovirus DNA in follicular bronchiectasis $(25 \%)$ was lower than expected. They speculated that this might be the consequence of a more effective immune elimination of the adenovirus in patients with a follicular histology, possibly as a consequence of local populations of antiviral cytotoxic lymphocytes. We speculate that one reason for the low prevalence of adenovirus in fibrotic lung disease is the elimination of adenovirus by the host's immune response, because the E1A region of adenovirus is responsible for sensitizing the infected cells to cytolysis by TNF [7], NK cells, and activated macrophages [8]. In lung tissues of patients with IPF and CVD-IP, macrophages and lymphocytes are activated, and produce proinflammatory cytokines. Another reason for the low prevalence may be a lower population of alveolar and bronchiolar epithelial cells where adenovirus persists, as demonstrated by ISH in the study by MATSUSE et al. [6], since these cells are damaged and replaced by fibrotic tissues.

The possible role of adenovirus, either in latent infection or in recurrent infection later in life, is open to 
question. The mechanism of its latency, a chronic, lowgrade infection, lysogenicity, or incorporation of the virus genome to the host cell, has not been determined. It is, therefore, possible that administration of corticosteroids might cause the virus to replicate. Corticosteroid treatment may suppress the function of NK cells and activated macrophages, or production of cytokines to eradicate the virus. The incidence of viral infection increases during administration of corticosteroids [15]. Therefore, the present study suggested the incidence of adenovirus infection may be increased, or a latent adenovirus infection may be reactivated in corticosteroidtreated patients. However, we should be careful to decide whether the lower values in the IPF and CVD-IP are statistically valid and biologically relevant and whether higher values in corticosteroid-treated patients are applicable, because the number of cases employed in this study was small.

Adenoviral infections are known to cause severe necrotizing bronchitis, bronchiolitis and bronchiolitis obliterans with associated pneumonic pulmonary fibrosis, both in animals [16] and in humans [17-20]. The E1A region of the adenovirus was used as the target DNA for amplification in this study because this region is responsible for transactivation of the viral and cellular genes, transformation of the cells, and the sensitization of infected cells to cytolysis by TNF [7], NK cells, and activated macrophages [8]. In contrast, a glycosylated 14,700 MW protein encoded by the E3 region of the adenovirus protects infected cells from lysis by TNF [21]. A glycosylated 19,000 MW protein encoded by E3 region is also thought to be involved in maintaining latent adenoviral infection [8]. This viral protein prevents cell surface expression of class I major histocompatibility complex (MHC) molecules by retaining them in the cell's endoplasmic reticulum. Failure of infected cells to express class I MHC protects them against destruction by cytotoxic T-cells, and allows the virus to persist in the host cell.

The present results suggest that corticosteroid treatment may be associated with the prevalence of adenovirus, although it is unlikely that adenovirus was aetiologically involved in the pathogenesis of IPF or CVD-IP. Because only one out of the eight cases of IPF or CVDIP that were positive for adenovirus E1A DNA by PCR, was positive for adenovirus DNA by ISH, and the population studied was small, additional studies are needed to demonstrate the expression of messenger ribonucleic acid (mRNA) or proteins of adenovirus in infected cells, in order to determine whether adenovirus is implicated in the pathogenesis of pulmonary fibrosis.

Acknowledgements: The authors thank S. Hayashi and J.C. Hogg for formalin-fixed, paraffin-embedded Graham 293 cells, Raji cells, and for lung tissue from patients with adenovirus pneumonia.

\section{References}

1. Li H, Gyllensten UB, Cui X, Saiki R, Erlich HA, Arnheim N. Amplification and analysis of DNA sequences in single human sperm and diploid cells. Nature 1988; 335: 414-417.

2. Kwok S, Sninsky JJ. Application of PCR to the detection of human infectious diseases. In: Erlich HA, ed. PCR
Technology: Principles and Applications for DNA Amplification. New York, Stockton Press, 1989; pp. 235-244.

3. Goelz SE, Hamilton SR, Vogelstein B. Purification of DNA from formaldehyde-fixed and paraffin-embedded human tissue. Biochem Biophys Res Commun 1985; 130: $118-126$.

4. Green M, Wold WSM, Mackey JK, Rigden P. Analysis of human tonsil and cancer DNAs and for RNAs or DNA sequence of group C (serotypes 1, 2, 5 and 6) human adenoviruses. Proc Natl Acad Sci USA 1979; 76: 6606-6610.

5. Horbath J, Palkonyay L, Weber J. Group C adenovirus DNA sequences in human lymphoid cells. $J$ Virol 1986; 59: 189-192.

6. Matsuse T, Hayashi S, Kuwano K, Keunecke H, Jeffries WA, Hogg JC. Latent adenoviral infection in the pathogenesis of chronic airways obstruction. Am Rev Respir Dis 1997; 146: 177-184.

7. Duerksen-Hughes P, Wold WSM, Gooding LR. Adenovirus E1A renders infected cells sensitive to cytolysis by tumor necrosis factor. J Immunol 1989; 143: 4193-4200.

8. Gooding LR, Wold WSM. Molecular mechanisms by which adenoviruses counteract antiviral immune defenses. Crit Rev Immunol 1990; 10: 53-71.

9. Albert J, Fenyö EM. Simple, sensitive, and specific detection of human immunodeficiency virus type 1 in clinical specimens by polymerase chain reaction with nested primers. J Clin Microbiol 1990; 28: 1560-1564.

10. Crystal RG, Fulmer JD, Roberts WC, Moss ML, Line BR, Reynolds HY. Idiopathic pulmonary fibrosis: clinical, histologic, radiographic, physiologic, scintigraphic, cytologic, and biochemical aspects. Ann Intern Med 1976; 85: 769-788.

11. Scharf SJ, Horn GT, Erlich HA. Direct cloning and sequence analysis of enzymatically-amplified genomic sequence. Science 1986; 33: 1076-1078.

12. Graham FL, Smiley J, Russell WC, Nairn R. Characteristics of a human cell line transformed by DNA from human adenovirus type 5. J Gen Virol 1977; 36: 59-72.

13. Fox JP. Viral contribution to chronic obstructive respiratory diseases. Yale J Biol Med 1968; 40: 484-492.

14. Bateman ED, Hayashi S, Kuwano K, Wilke TA, Hogg JC. Latent adenoviral infection in follicular bronchiectasis. Am J Respir Crit Care Med 1995; 151: 170-176.

15. Ginzler E, Diamond H, Kaplan D, Weiner M, Schlesinger M, Seleznick M. Computer analysis of factors influencing frequency of infection in systemic lupus erythematosus. Arthritis Rheum 1978; 21: 37-44.

16. Castleman WL. Bronchiolitis obliterans and pneumonia induced in young dogs by experimental adenovirus infection. Am J Pathol 1985; 119: 495-504.

17. Chany C, Lépine P, Lelong M, Vinh LT, Satgé P, Virat J. Severe and fatal pneumonia in infants and young children associated with adenovirus infections. Am J Hyg 1958; 67: 367-378.

18. Becroft DMO. Histopathology of fatal adenovirus infection of the respiratory tract of young children. J Clin Pathol 1967; 20: 561-569.

19. Lang WR, Howden CW, Laws J, Burton JF. Bronchopneumonia with serious sequelae in children with evidence of adenovirus type 21 infection. Br Med J 1969; 1: 73-79.

20. James AG, Lang WR, Liang AY, et al. Adenovirus type 21 bronchopneumonia in infants and young children. $J$ Pediatr 1979; 95: 530-533.

21. Gooding LR, Elmore LW, Tollefson AE, Brady HA, Wold WSM. A 14,700 MW protein from the E3 region of adenovirus inhibits cytolysis by tumor necrosis factor. Cell 1988; 53: 341-346. 